\title{
Forgery Detection in Meteorological Facsimile Maps via Intrinsic Features
}

\author{
Wang Xiaotong, Xu Guanlei, YangDa* \\ Navigation\&Ocean Department of Dalian Navy Academy \\ Dalian, China \\ *Yangda1977@163.com
}

\author{
Ma Yue \\ Navigation Department of Dalian Navy Academy \\ Dalian, China
}

\begin{abstract}
This paper discusses the forgery detection problem in meteorological facsimile maps via the intrinsic features of these maps. Meteorological facsimile maps are of much importance to navigation in ocean. Once the meteorological facsimile maps are tampered, the safety of ships will be possibly greatly decreased because of possible disaster by execrable weather. We exploited the problem to detect the possible forgeries by the intrinsic features of meteorological facsimile maps such as binary value, fixed form, sparseness and rationallydeveloping weather system and so on. Experiments show that the forgeries can be detected effectively in meteorological facsimile maps via the proposed intrinsic features.
\end{abstract}

Index Terms-image forgery detection; meteorological facsimile map (MFM); intrinsic feature.

\section{INTRODUCTION}

Due to fast growth of Internet applications and digital equipments, digitized images become more and more popular. Because of the ease of digital duplication and tampering, image security becomes an important issue nowadays [1,6-9]. In certain application cases, it is a risk if a set of secret image is held by only one person without extra copies because the secret image set may be lost incidentally or modified intentionally. In some other cases, it might be necessary for a group of persons to share a certain set of secret data. In particular, how to share a secret image has attracted wide attention in recent years because of the popular uses of images in network applications or other fields.

Meteorological navigation is of much importance to the ships. Recently, the meteorologic electrographs have been widely used in all kinds of ships [2], and the general hydrometeorological information can be gotten via the meteorologic electrographs. The meteorological facsimile map (MFM) is the graphic form of numerical value forecast, and consequently, navigation masters can obtain the useful meteorological information directly via these MFMs. However, in different ocean zones, different weathers and different settings of electromagnetic wave and etc., many MFMs contain noise and distortion (even if the forgeries), sometimes, intentional forgeries especially in war time. On one hand, the meteorological information obtained by navigation masters through MFMs mainly depend on the ability of these navigation masters, therefore, the noise or the people that intentionally tamper the maps greatly disturbs the navigation masters. Therefore, before employment, we must know whether these maps are tampered or not. Up till now, there has been no published paper covering this problem on MFM.

MFMs have special properties [2,4]. Firstly, MFMs have the representative form of vector maps, however, they are binary-valued maps in fact. Secondly, MFMs have special given forms, and their bottoms are geographical information (e.g., contours of islands and the mainland, called as bottommap), and their top levels are all kinds of meteorological marks and notations (e.g., atmospheric pressure lines and iceberg and etc.), in addition, these meteorological marks and notations have prescriptive and standard forms. Thirdly, nearly all the weather systems have the consecutive features, or in other words, these weather systems must change and move consecutively. If above features are destroyed, it is rational to oppugn the truth of the MFMs so that one can strive to find forgeries. This paper will discuss the forgery detection in MFMs.

\section{INTRINSIC FEATURES OF MFMS FOR FORGERY DETECTION}

In this section, we will discuss the intrinsic features in MFMs in great details, which is of much importance to the forgery detection. For example, there are a lot of standard marks, and these marks will not change with the changing of different meteorologic electrographs, e.g., these marks such as " $\mathrm{H}$ ", " $\mathrm{L}$ " " $\oplus$ " and these numbers " $1,2,3,4,5,6,7,8,9,0$ " and so on. Therefore, we know that MFMs mainly have the following intrinsic features.

(1) MFMs are binary-valued dot-pixel maps. In MFMs that are free of any noises there are only two values ( 0 and 255 for 256-level gray images). Generally, there are no other values. Otherwise, we can view these pixels that are not 0 or 255 as noises.

(2) In MFMs, there is a fixed form that we call as bottom map, i.e., every piece of MFM has a fixed geographical zone and contains the fixed geographical information, e.g., contours of islands and the mainland, longitude lines and latitude lines and so on. MFMs contain bottom maps.

(3) In MFMs, there are a lot of fixed-formed meteorologic symbols and marks, which are standard forms. Once the type of MFM is determined, the forms of these meteorologic symbols and marks are known.

(4) In MFMs, the main information carriers are all kinds of lines or curves. The meteorologic symbols and marks, 
which are also all kinds of curves with width of a few pixels, can be taken as all kinds of lines or curves. MFMs can be taken as pseudo-vector maps.

(5) In MFMs, the blank zones (255 for blank and 0 for nonblank) cover over $60 \%$ of the whole acreage, which is verified by a lot of experiments. Hence, MFMs are sparse.

(6) All the weather systems have the consecutive features, or in other words, these weather systems change and move consecutively without jumping over wide gaps.

(7) All the weather systems' development follows physical rules of weather forecast.

These intrinsic features in above also can be concluded as three main features: binary-valued, fixed-formed and rational, which is very helpful for the forgery detection. In the following sections, we will use these intrinsic features to detect the potential or possible forgery.

\section{BOTTOM MAP CREATION}

In different ocean zones, according to current time and loaded frequencies one can know the type of the MFM. Once the type of the MFM is known, we can know the approximate type of the bottom map. However, in ships since the meteorologic electrographs may have different performances and working states, the bottom maps are not the same for all the ships. Hence, in different ships the bottom maps should be created separately. In some paper [3], they adopt the handdrawing to obtain the bottom map, which is slow and errors will come into being very easily.

From the intrinsic feature (2) of MFMs in above section 2, one simple but very efficient method to create the bottom map is the employment of the same type MFMs in different time because they contain the same bottom map despite of the different meteorologic information in form of same meteorologic symbols and marks at different geographical locations.

For the same type of MFMs $f_{1}(x, y), \cdots, f_{l}(x, y), \cdots, f_{L}(x, y)$ $(l=1, \cdots, L)$, we have

$$
f_{\text {avg }}(x, y)=\frac{1}{L} \sum_{l=1}^{L} f_{l}(x, y) .
$$

Since

$$
f_{l}(x, y)=f_{g, l}(x, y)+f_{d}(x, y)(l=1, \cdots, L),
$$

where $f_{d}(x, y)$ is the bottom map, $f_{g, l}(x, y)$ is the map that contains all the information of the MFM except for the bottom map. Clearly, for different $l, f_{g, l}(x, y)$ is different. Thus

$$
f_{\text {avg }}(x, y)=\frac{1}{L} \sum_{l=1}^{L} f_{g, l}(x, y)+f_{d}(x, y) \text {. }
$$

From the sparse feature (5) of MFMs, generally the locations of $f_{g, l_{1}}(x, y)$ are different with that of $f_{g, l_{2}}(x, y) \quad\left(l_{1} \neq l_{2}\right)$. Therefore, ${ }^{g(x, y)=\frac{1}{L} \sum_{l=1}^{L} f_{g, l}(x, y)}$ will contain a lot of pixels that are more than 0 and less than 255 . In addition, with the increasing of $L$, the more and more of these pixels are. Finally, we obtain

$$
f_{\text {avg }}(x, y)=g(x, y)+f_{d}(x, y) \text { with } f_{d}(x, y)=0 \text { or }
$$
$f_{d}(x, y)=255$ and $0<g(x, y)<255$.
Therefore, we can obtain the bottom map $f_{d}(x, y)$ :

$$
f_{d}(x, y)=\left\{\begin{array}{l}
0 \quad \text { if } f_{\text {avg }}(x, y)<t d \\
255 \quad \text { else }
\end{array} .\right.
$$

Generally, we take the value $t d=255 / L$. Since there are some isolated black dots or speckles in the map after performing Eq.(5), we adopt a acreage operator $H_{a c}($ ), which denotes as

$$
H_{a c}(Z(x, y))=\left\{\begin{array}{lc}
1 & \operatorname{num}(Z(x, y))<5 \\
Z(x, y) & \text { else }
\end{array},\right.
$$

where $Z(x, y)$ denotes a block centered in $(x, y)$, num( $)$ is the counter of zeros. Thus the bottom map is optimized. Clearly, this method is robust in the presence of noises.
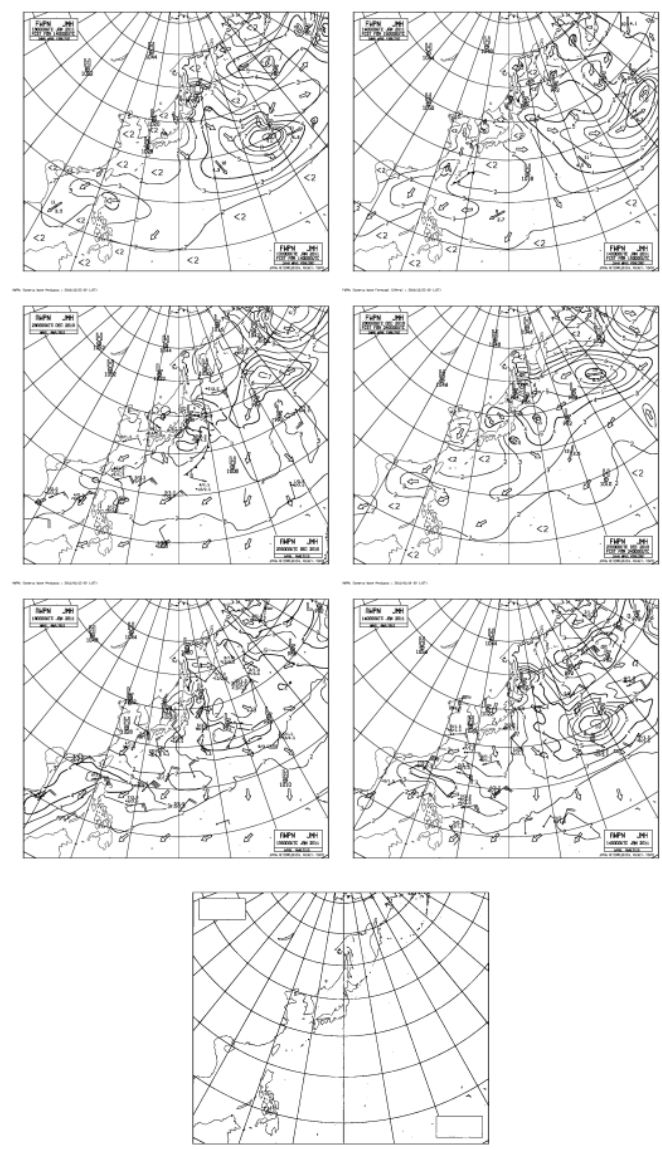

Fig.1. Bottom map creation. The former 6 maps are different MFMs, and the final one is the created bottom map from them by us.

\section{FORGERY DETECTION}

In this paper the forgery detection will mainly focus on the two features defined in (6) and (7). Through the rules of MFMs, one can easily detect the forgeries.

\section{A. Bottom map removal}

Before forgery detection, the removal of bottom map removal is our first work so that the later processing can be done without the influence of bottom maps.

Then we obtain 


$$
f_{\text {con }}(x, y)=f_{n}(x, y)-f_{d}(x, y)
$$

Clearly, $f_{c o n}(x, y)$ contains all the useful hydrometeorological information $f_{g}(x, y)$ and possible tampered information. Eq.(8) needs that $f_{n}(x, y)$ and $f_{d}(x, y)$ matches, otherwise, more errors will be introduced. Generally, if we adopt the matching method defined in [5], one can obtain the satisfied result.

\section{B. The main possible forgeries}

In forgery detection, one must know where the forgery often occurs in MFM. After a lot of reviewing, we find that there are some positions in MFM where the forgery often occurs as shown in fig.2. In this paper, we will mainly discuss three aspects that are possible to be tampered.

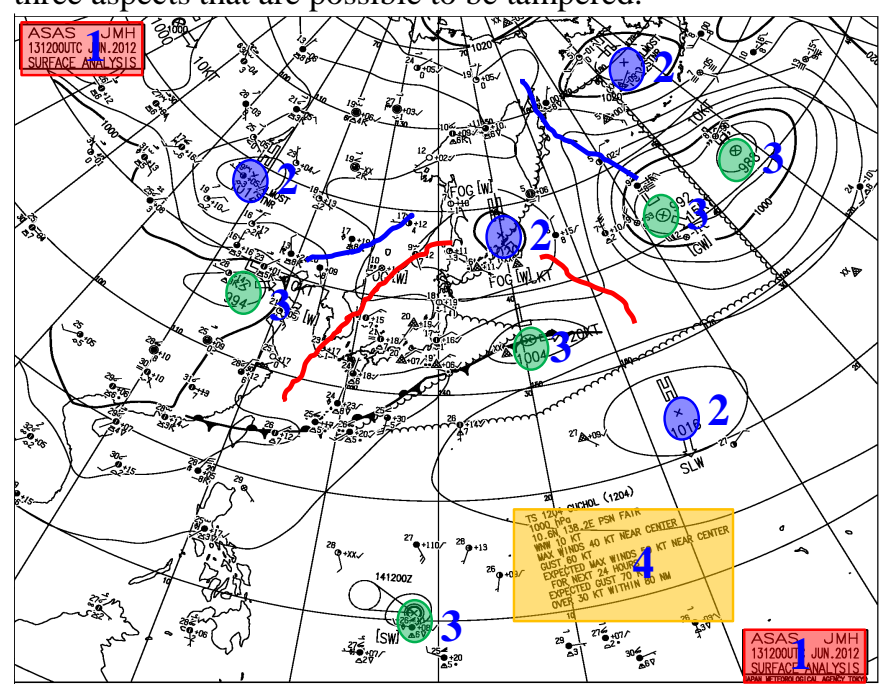

Fig.2. The possible forgeries in one MFM (shown in colored zones).

(1)Time forgery: this type of forgery is mainly for the time tampering. If one changes the time in a MFM, then this MFM will greatly disturb the judgement what to do in the next step. This type of forgery mainly occurs in two regions in MFMs: the title zone and the bottom title zone shown by blue word 1 . In order to judge whether there is forgery or not, one must compare this MFM with the adjacent MFMs so that see whether this time is true or not.

(2)Air pressure forgery: this type of forgery is mainly for the value tampering. If one changes the value in a MFM, then this MFM will greatly disturb the judgement what extent the weather system will be. This type of forgery can occur in anywhere the weather system centers occupy such as the circle zones shown in fig. 2 by word 2 and 3. In order to judge whether there is forgery or not, one must compare all the pressure lines in this MFM so that see whether center air pressure is tampered or not. Generally, the high pressure center and low pressure center will occur every two systems or in the same two high (low) pressure systems there should be a low (high) ridge line. The low (high) ridge lines are shown in fig. 2 with blue (red) colors respectively.

(3)Explanation forgery: this type of forgery is mainly for the explanation tampering in words such as typhoon explanation. If one changes the explanation in a MFM, then this MFM will greatly disturb the judgement what extent the tropical low pressure system will be. This type of forgery only occurs in south sea zones such as the blank zones shown in fig. 2 by word 4 . In order to judge whether there is forgery or not, one must compare all the pressure lines near the low pressure center in this MFM so that see whether the explanation is tampered or not. Generally, the development of the tropical low pressure systems follow some rules such as the moving ways will be four types: to west, to north-west, to north-west first and then turn to north-east and so on. In other words, all the weather systems' development follows physical rules of weather forecast.

\section{Experiments}

In this section, we will use some MFMs to test the main forgeries to show the influences by them. We use 10 MFMs shown in fig. 3 to test some persons (two teams of persons) that are familiar with the knowledge of MFMs and the intrinsic features. In every MFM, we don't tell whether the MFM is tampered or not. At first, we let the first team's persons (Team 1) to detect the forgeries without telling them to use the intrinsic features. Then we let the second team's persons (Team 2) to detect the forgeries with telling them to use the intrinsic features. Table 1 is the forgery detection result by the two teams respectively.

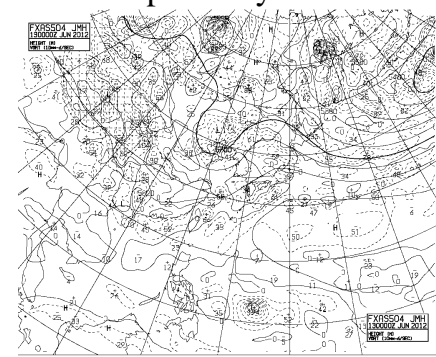

(a)

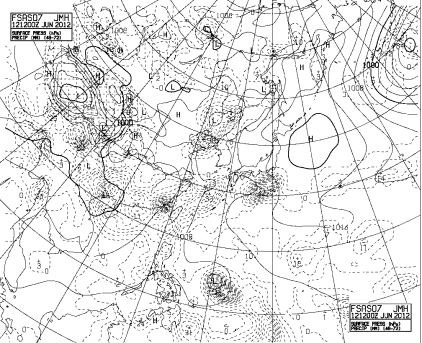

(b)

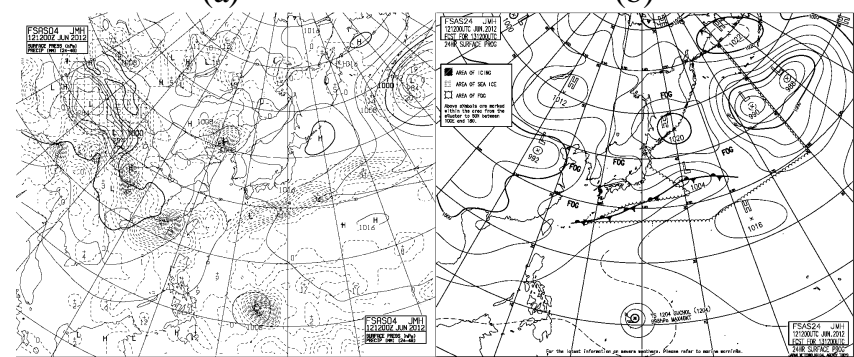

(c)

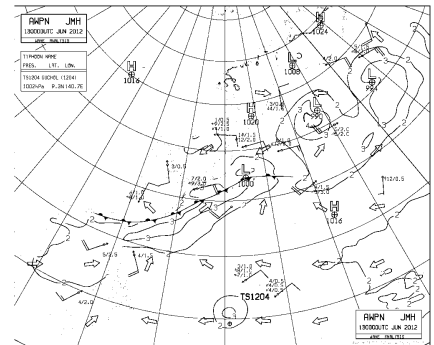

(e) (d)

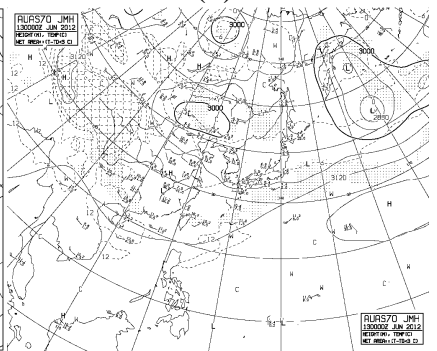

(f) 


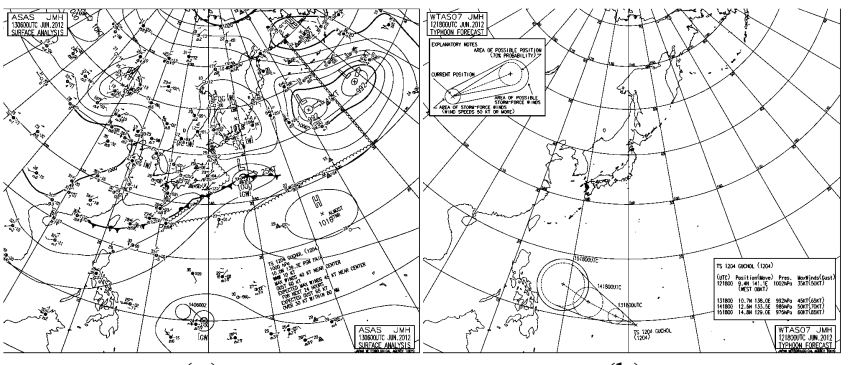

(g)

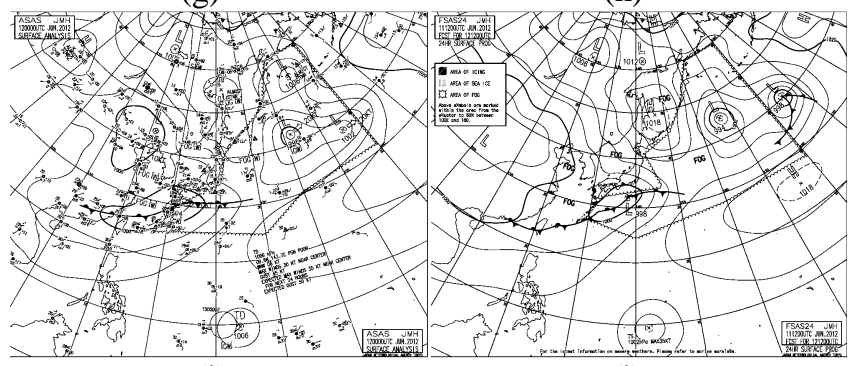

(i)

Fig.3. The tested MFMs.

TABLE I. THE COMPARISON OF THE TWO TEAMS WITH AND WITHOUT KNOWING THE INTRINSIC FEATURES.

\begin{tabular}{|c|c|c|c|c|c|c|c|c|c|c|}
\hline & \multicolumn{10}{|c|}{ The forgery detection rate (\%) } \\
\hline & \multicolumn{10}{|c|}{ Fig.3 } \\
\hline & (a) & (b) & (c) & (d) & (e) & (f) & (g) & (h) & (i) & (j) \\
\hline & 50 & 40 & 45 & 55 & 55 & 50 & 50 & 40 & 45 & 45 \\
\hline 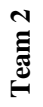 & 85 & 95 & 90 & 95 & 85 & 90 & 90 & 95 & 90 & 95 \\
\hline
\end{tabular}

Clearly, via the intrinsic features of MFMs, one can easily find the forgery, especially the obvious tampering in maps. After training by the intrinsic features of MFMs, it is possible that a laypeople can also detect effectively the forgeries in MFMs with the detection rate over $80 \%$.

\section{CONCLUSIONS}

Meteorological facsimile maps are of much importance to navigation in ocean. Once the meteorological facsimile maps

are tampered, the safety of ships will be possibly greatly decreased because of possible disaster by execrable weather. This paper discusses the forgery detection problem in meteorological facsimile maps via the intrinsic features of these maps. We find that it is possible if one wants to detect the possible forgeries by the intrinsic features of meteorological facsimile maps such as binary value, fixed form, sparseness and rationally-developing weather system and so on. The final experiments show that the forgeries can be detected effectively in meteorological facsimile maps via the proposed intrinsic features if the persons know these intrinsic features well.

\section{ACKNOWLEDGMENT}

This work is supported by NSFCs (61002052 and 61250006).

\section{REFERENCES}

[1] C.R. Gonzalez, E. Richard Woods, Digital Image Processing, second ed., Pearson Education, 2003.

[2] Zhang Yongning. Meteorology for Mariners, Dalian Marine University Press, Dalian, 2008.

[3] $\mathrm{Hu}$ X.B, Study on the vectorization technology of dot matrix meteorological facsimile maps [dissert], 2007, Harbin Engineering University.

[4] Zhou Lijia, Qian Zhibo, Xu Guanlei, Binary Quantization based Noise Removal for Meteorological Facsimile Maps, 2011 4th IEEE International Conference on Computer Science and Information Technology, vol.03,45-48.

[5] Li Bo, Wang Xiaotong, Yang Changqing, Jin Liang'an, “Threepoint locally adaptive searching in gray scale projection algorithm for electronic image stabilization," Opto-electronic Engineering, 2004,09, 69-72.

[6] Verheul, E.R., van Tilborg, H.C.A., 2007. Construction and properties of $\mathrm{k}$ out of $\mathrm{n}$ visual secret sharing schemes. Designs, Codes, Cryptography 11, 179-196.

[7] Wu, D.C., Tsai, W.H., 1998. Data hiding in images via multiplenumber conversion and lossy compression. IEEE Transactions Consumer Electronics 44 (4), 1406-1412.

[8] Wu, D.C., Tsai, W.H., 1999. Embedding of any type of data in based on a human visual model and multiple-based conversion. Pattern Recognition Letters 20, 1511-1517.

[9] Wang XiaoTong, Shao ChengYong, Xu XiaoGang, Niu XiaMu. Lossless data hiding of $2 \mathrm{D}$ vector maps based on difference expansion. IEEE Transactions on Information Forensics and Security. 2007.12. 